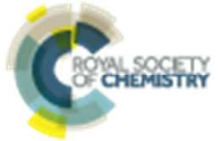

Nanoscale

\title{
The duo roles of functional groups in the photoluminescence of graphene quantum dots
}

\begin{tabular}{|r|l|}
\hline Journal: & Nanoscale \\
\hline Manuscript ID & NR-ART-10-2015-007042.R1 \\
\hline Article Type: & Paper \\
\hline Date Submitted by the Author: & 06-Dec-2015 \\
\hline Complete List of Authors: & $\begin{array}{l}\text { Wang, Shujun; Griffith University, Queensland Micro- and Nanotechnology } \\
\text { Centre; Griffith University, Environmental Engineering } \\
\text { cole, ivan; CSIRO, Materials Science and Engineering } \\
\text { Zhao, Dongyuan; Fudan University, Department of Chemistry } \\
\text { Li, Qin; Griffith University, Queensland Micro- and Nanotechnology Centre; } \\
\text { Griffith University, Environmental Engineering }\end{array}$ \\
\hline
\end{tabular}




\title{
ARTICLE
}

Cite this: DOI: $10.1039 /$ xoxxooooox

\section{The duo roles of functional groups in the photoluminescence of graphene quantum dots}

\author{
Shujun Wang ${ }^{a b}$, Ivan S. Cole ${ }^{c}$, Dongyuan Zhao ${ }^{d}$, and Qin $\mathrm{Li}^{\mathrm{ab} *}$
}

Received ooth January 2012, Accepted ooth January 2012

DOI: 10.1039/xoxxooooox

www.rsc.org/
The photoluminescent properties of graphene nanoparticle (named graphene quantum dots) have attracted significant research attention in recent years owing to their profound application potential. However, the photoluminescence (PL) origin of this class of nanocarbons is still unclear. In this paper, combining direct experimental evidences enabled by a facile sizetunable oxygenated graphene quantum dots (GQDs) synthesis method and theoretical calculations, the roles of aromatic core, functional groups and disordered structures (i.e. defects and $\mathrm{sp}^{3}$ carbon) in the PL of oxygenated GQDs are elucidated in detail. In particular, we found that the functional groups on GQDs play duo roles in the overall emission: 1) they enable $\pi^{*} \rightarrow \mathrm{n}$ and $\sigma^{*} \rightarrow \mathrm{n}$ transitions, resulting in molecular type of PL, spectrally invariable with change of particle size or excitation energy; 2) similar to defects and $\mathrm{sp}^{3}$ carbon, functional groups also induce structural deformation to the aromatic core, leading to mid-gap states or in other words, energy traps, causing $\pi^{*} \rightarrow$ mid-gap states $\rightarrow \pi$ transitions. Therefore, functional groups contribute to both the blue edge and the red shoulder of GQDs' PL spectra. The new insights on the role of functional groups in PL of fluorescent nanocarbons will enable better designs of this new class of materials.

\section{Introduction}

Graphene quantum dots (GQDs), a nano version (typically less than $100 \mathrm{~nm}$ in lateral dimension) of graphene whose interesting properties ${ }^{1-5}$ distinguish them from bulk graphite, have recently received significant scientific attention. The attractive photoluminescence (PL) properties of GQDs place them amongst the emerging optical materials for advanced applications such as bio-imaging, light emitting devices and optical sensing ${ }^{6,7}$. Most of the GQDs synthesized by far contain oxygen functional groups and disordered structures $^{8-13}$. However, despite of the successes achieved in preparing various types of oxygenated GQDs, the fundamental question on the origin of PL from such GQDs still requires further understanding.

A well-known feature of graphene is the absence of band gap in its band structure. However, for graphene quantum dots, the quantum confinement effect induces size dependent band gap ${ }^{14}$, 15. Hence, one plausible mechanism for the PL of oxygenated GQDs is the size dependent band gap induced by the quantum confinement effect in which the energy gap between bonding $(\pi)$ and anti-bonding $\left(\pi^{*}\right)$ increases with the reduction of dot size $e^{10}$, ${ }^{16}$. Other proposed mechanisms including the energy traps ${ }^{9,17}$, combined intrinsic and defects emission ${ }^{18,19}$, suggest the involvement of functional groups or defects in the PL of oxygenated GQDs. Moreover, an analogy was often drawn between oxygenated GQDs and graphite oxide (GO, having similar PL with GQDs) ${ }^{16,20-22}$. Although a general consensus is that the PL of GQDs might be a combined effect of aromatic core (i.e $\mathrm{sp}^{2}$ skeleton), functional groups and disordered structures (i.e. defects and $\mathrm{sp}^{3}$ carbon) ${ }^{6,23}$, the specific roles of these contributing factors, particularly with regards to their fingerprints on static and time-resolved PL spectra, are not yet resolved. A major hindrance for elucidating the PL mechanism is to produce oxygenated GQDs with well-controlled size while retaining the similarity in chemical composition. ${ }^{9,10,16-19}$

Herewith, we first introduce a synthesis method that can quasi-continuously tune the GQDs size while retaining chemical structural similarity, which forms the basis of the following investigations. We then elucidate the PL mechanisms by a series of static and dynamic PL spectral analysis for both as-synthesized GQDs and chemically reduced GQDs. The PL mechanisms as revealed by the spectral analysis were further confirmed with the PL pH-dependent behaviour. Furthermore, first principle calculations include density functional theory (DFT) and Maximal Localized Wannier Functions (MLWF) were performed, which provides in-depth understanding on the energy levels introduced by aromatic core, functional groups and structural defects. In particular, we found that the functional groups on GQDs play duo roles in the overall 
emission: 1) they enable $\pi^{*} \rightarrow \mathrm{n}$ and $\sigma^{*} \rightarrow \mathrm{n}$ transitions, resulting in molecular type of PL, spectrally invariable with the change of particle size or excitation energy, which often appear on the blue side of the PL spectra; 2) similar to defects and $\mathrm{sp}^{3}$ carbon, they induce structural deformation, leading to mid-gap states or in other words, energy traps, causing $\pi^{*} \rightarrow$ mid-gap states $\rightarrow \pi$ transitions, which are often exhibited as the broadened PL spectra extending into longer wavelength.

\section{Experimental}

\subsection{Synthesis of GQDs}

The GQDs were prepared from carbon black. For a typical procedure, $50 \mathrm{mg}$ of carbon black (Spuer P, Alfa Aesar) was put inside a $50-\mathrm{ml}$ round flask. $6 \mathrm{ml}$ of concentrated $\mathrm{HNO}_{3}$ ( $70 \%$, Chem-Supply) was then introduced to the round flask followed by adding $18 \mathrm{ml}$ of $\mathrm{H}_{2} \mathrm{SO}_{4}$ (98\%, Chem-Supply) slowly and carefully under magnetic stirring (350 rpm). Upon the completion of adding the chemicals, the round flask was placed into a silicon oil bath with temperature set as $160^{\circ} \mathrm{C}$. A condenser was subsequently applied to reflux the vapourized acids and acids decompositions (majorly $\mathrm{HNO}_{3}$ decompositions since gas with brown colour were generated inside round flask) and the reflux was quite critical as without it nitric acid would fully decompose soon resulting in termination of reaction. Upon completion of reaction for targeted duration, the reacted materials were poured slowly into $200 \mathrm{~mL}$ of DI water under vigorous stirring. The solution was then neutralized to $\mathrm{pH} 7$ by adding $\mathrm{NaOH}$ (Chem-Supply). The final solution was subsequently concentrated to $100 \mathrm{ml}$ before subjected to dialysis with a 500Da tube membrane (SpectrumLabs) for 3 days. For a typical reduction test, $10 \mathrm{~mL}$ of as-synthesized GQD solution (for G20, G80 and G210) was added to a glass vial followed by addition of $10 \mu \mathrm{L}$ of hydrazine $(35 \%$, Acros Organics) solution. The vial was then placed into an oil bath with temperature kept at $95{ }^{\circ} \mathrm{C}$ for 1 hour.

\subsection{Characterization}

An UV/Vis spectrometer (Agilent 8453) was applied for acquisition of the absorption spectra. Fluorescent spectra and Photoluminescence excitation spectra were collected through a fluorescence spectrometer (Thermo Scientific Lumina). Fluorescent lifetime was recorded on another fluorescence spectrometer (Edinburgh Photonics FLS920) with 372nm and $433 \mathrm{~nm}$ pulse laser excitation sources. Different chemical bonding conditions were qualitatively evaluated through a Fourier transformation infrared (FTIR) spectrometer (Spectrum Two, Perkin Elmer). Elemental compositions and chemical bonding status were quantitatively analysed by X-ray photoelectron spectroscopy (XPS, Kratos Axis Ultra). Highresolution transmission electron microscope (HRTEM, Philips Tencai F20), along with atomic force microscope (AFM, NTMDT NTEGRA Spectra) were adopted for the morphology analysis.

\subsection{Theoretical calculation}

The density functional theory (DFT) calculations were conducted with the ABINIT codes (Version 7.4.3). Localized density approximation (LDA) was adopted for the exchangecorrelation functional. Norm-conserving pseudo-potentials of
Troullier-Martins type were applied. Proper cut-off energies and super cell sizes (see Supporting Information) were chosen via convergence studies. Each model was relaxed via the Broyden-Fletcher-Goldfarb-Shanno minimization (BFGS) process. The Maximal Localized Wannier Functions (MLWF) were extracted by Wannier90 (used as a library of the ABINIT codes) for the three 7 rings GQD models. More details of the calculation could be found in the supplementary information.

\section{Results and discussion}

\subsection{Photoluminescence of as synthesized GQDs}

Like most of GQDs synthesized by far, the as synthesized GQDs (Fig. S1) in this study possess excitation dependent photoluminescence (PL) as shown in Figure 1a, namely the maxima of PL spectra shifts with change of excitation energy. In addition to the excitation dependence, another feature is that the PL maxima present a blue-shift trend with the extension of reaction duration from $\mathrm{G} 20$ to $\mathrm{G} 210$. Moreover, when all the as-synthesized GQDs are excited under a fixed photon energy, the maxima of the static PL spectra also present a gradual blue shift trend (Fig. 1 b). In contrast to the excitation dependence and blue shift of the spectral maxima of PL, two shoulder peaks at $390 \mathrm{~nm}$ and $440 \mathrm{~nm}$ respectively which only responses to high energy excitation (i.e. excitation wavelength $<400 \mathrm{~nm}$ ) do not shift with the excitation energy (As marked by in Fig. 1 a) and it is also independent to the change of reaction duration (as marked in Fig. 1 b). The variable PL maxima and invariable shoulder peaks suggests that multiple emissive mechanisms should be responsible for the overall PL emission of as synthesized GQDs.

The as synthesized GQDs contain similar types of oxygenated functional groups including hydroxyl (-OH), epoxy $(-\mathrm{O}-)$, carbonyl $(-\mathrm{C}=\mathrm{O})$ and carboxyl $(-\mathrm{COOH})$ as revealed by the XPS and FTIR characterization (Fig.S7 and Fig.S8). Moreover, as the reaction duration extended from $20 \mathrm{~min}$ (for G20) to $210 \mathrm{~min}$ (for G210), the change of their atomic concentrations of any individual functional groups is random (Fig. S7 and Table S1) with no specific trend, however, the carbon to oxygen ratios ( $\mathrm{C} / \mathrm{O}$ ration) are all around 3 (Fig. $1 \mathrm{c}$ and Table S1), which suggests there might be interconversion among the oxygen functional groups (e.g. converting $\mathrm{C}-\mathrm{O}$ to $\mathrm{C}=\mathrm{O}$ ) with extended reaction duration, but all the as synthesized GQDs maintain similar degree of oxidation. Hence, the gradual blue shift phenomenon of the PL spectra is unlikely due to the change of oxygen functional groups. However, with the extension of reaction time, the particle size of the GQDs decreased gradually (Fig. $1 \mathrm{c}$, and more details in supplementary information on particle size measurements via Raman, DLS and TEM). Therefore, it is highly likely that the blue shift of PL maxima is an indication of the quantum confinement feature for which the smaller the size of GQDs, the wider the bandgap of the GQDs, the higher the emission energy is ${ }^{18}$. We believe an edge-etching mechanism which is capable of continuously removing the edge atoms of GQDs as illustrated by Figure S9 in Supporting Information is the reason behind the similarity of degree of oxidation and the gradual reduction of particle size. The blue shift of the PL spectra maxima is in good agreement with the blue shift of PLE spectra (Fig. S10) and the migrating bands as revealed from fitting of the UV/Vis spectra (Fig. S11) of the as synthesized GQDs.

As for the shoulder peaks independent of excitation energy and particle sizes, they should originate from another emissive site which possesses fixed energy gaps. Hence, the static PL 


\section{ARTICLE}

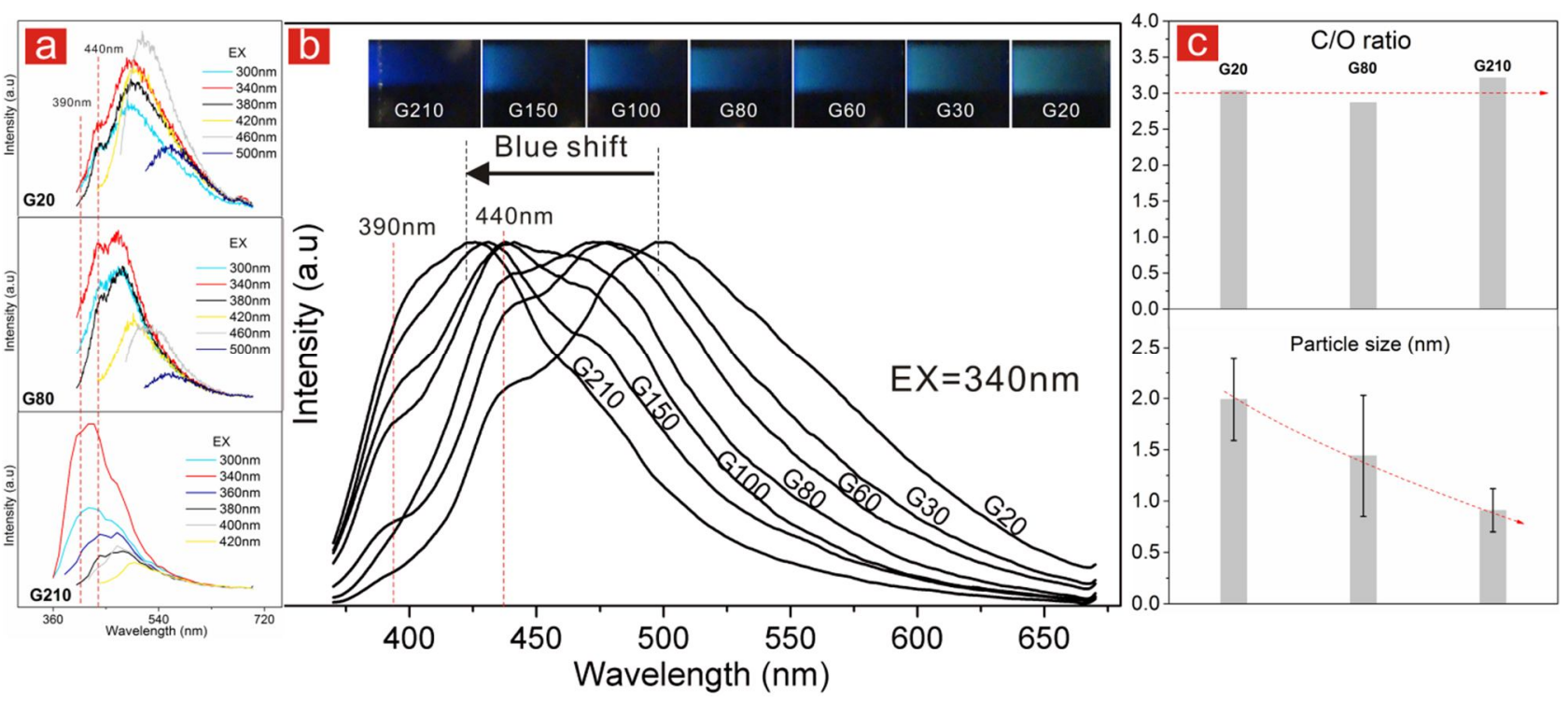

Figure 1. PL of as synthesized GQDs: under multiple excitations for G20, G80, G210 (a) and under 340nm excitation for all the GQDs (b); C/O ratio from XPS measurement and particle size from DSL (c)

spectra suggest multiple emissive sites for GQDs; while some possess fixed energy gap contributing to emissions which does not change position with varying excitation energy or particle size, others present quantum confinement feature showing excitation dependence and blue shift upon increasing reaction duration.

For a fluorophore having different possible emissive mechanisms, time-correlated single photon counting (TCSPC) is a useful tool that could identify each individual component and also provides the associated fluorescent lifetime and weight of each lifetime component. For this purpose, two laser excitation sources $377 \mathrm{~nm}$ and $443 \mathrm{~nm}$ were applied for the TCSPC measurements. For each excitation, the TCSPC spectra were collected at different emission wavelength throughout the static PL spectra. In order to reveal the possible oxygen related emission components from the rest, the TCSPC spectra of the chemically reduced GQDs (i.e. rG20, and rG210) that were prepared by hydrazine treatment of the two as synthesized GQDs (i.e. G20, and G210) were also measured. Similar to most of the literatures ${ }^{10,24}$ of oxygenated GQDs, all of the TCSPC decay curves can be well fitted with a tri-exponential function (see supplementary information). In spite of the differences in excitation and emission energy, all of the triexponential fittings comprise a fast component $\left(\tau_{1}\right)$ with a lifetime constant around $1 \mathrm{~ns}$, an intermediate component $\left(\tau_{2}\right)$ of $3 \sim 4$ ns and a slow component $\left(\tau_{3}\right)$ of $10 \sim 14 \mathrm{~ns}$, which suggests that there are possibly three emissive sites contributing to the PL of the as-synthesized GQDs.

Figure 2 a $\sim \mathrm{c}$ summarises the weight of each lifetime component for G20 and its chemically reduced counterpart rG20 at different emission wavelengths (enabled by monochromic filters) under $377 \mathrm{~nm}$ excitation (see Figure S13 in supplementary information for a complete summary of the weights of lifetime components for G20, rG20, G210 and rG210 under both $377 \mathrm{~nm}$ and $443 \mathrm{~nm}$ excitation). After reduction, the percentage of the fast component $\tau_{1}$ decreased towards the total photon counts (Fig. 2 a and Fig. S13), whereas the percentage of the slow component $\tau_{3}$ increased at both excitation energies and at all probed emission positions (Fig. 2 $\mathrm{c}$ and Fig. S13). The intermediate component $\tau_{2}$ does not exhibit obvious change in its percentage before and after chemical reduction.

Previously, the fast emission component of oxygenated GQDs has been ascribed to $\pi \rightarrow \pi^{*}$ transition, and the slow ones are considered to be related to the oxygen-containing functional groups ${ }^{24}$. However, our PL lifetime analysis shows that it is highly likely that $\tau_{1}$, the fast emission component, is associated with the oxygen functional groups which might contribute to the PL through electron transitions such as $n-\sigma^{*}$ (for C-O-C and $-\mathrm{OH}$ ) and $n-\pi^{*}$ (for $\mathrm{C}=\mathrm{O}$ and $\mathrm{COOH}$ ), and the decreased percentage of $\tau_{1}$ in total photon counts is a result of reduction in oxygen content as indicated by the increase of $\mathrm{C} / \mathrm{O}$ ratio post reduction (Fig. 2 d). It is also observed that the weight of $\tau_{1}$ decreases with the red shifting of emission positions, which indicates that the emission related to oxygenated functional groups most likely reside on the blue side of the PL spectra. Therefore, $\tau_{1}$ should correspond to the spectrally invariable shoulder peaks (i.e. $390 \mathrm{~nm}$ and 440nm emissions) observed on the static PL spectra as shown in Figure $1 \mathrm{a}$ and $\mathrm{b}$.

The percentages of $\tau_{2}(3-4 \mathrm{~ns})$ is similar before and after the hydrazine reduction (Fig. 2 b). Nevertheless, for most of the tested samples, the weight of $\tau_{2}$ shows a trend increases firstly to a peak value then drop (Fig. 2 b and S13) with the red shifting of the emission positions, which coincides with the 


\section{ARTICLE}
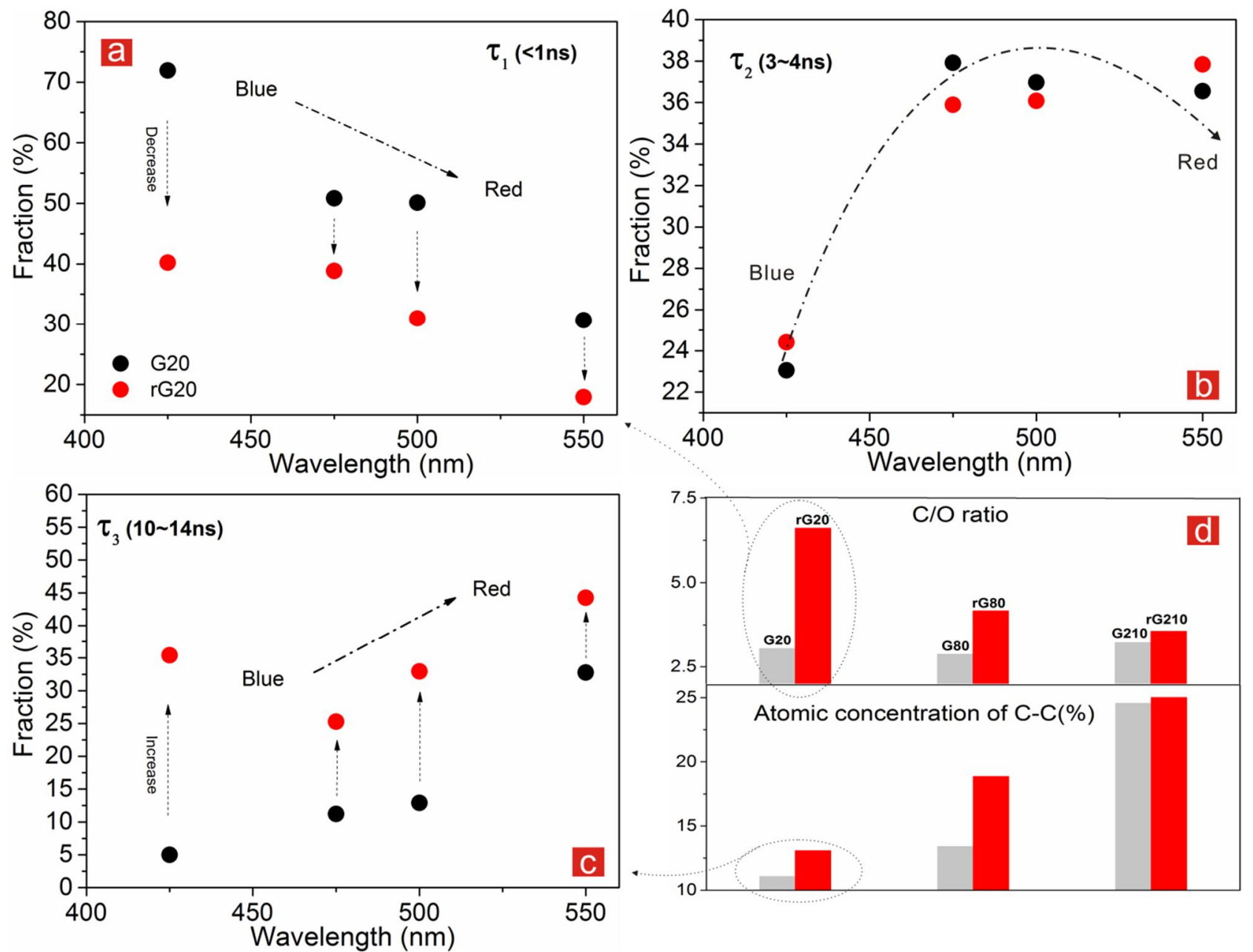

Figure 2. PL lifetime components of G20 from TCSPC measurement (a-c) and $\mathrm{C} / \mathrm{O}$ ratio and atomic concentration of aliphatic carbon $\left(\mathrm{sp}^{3}\right.$ carbon) from XPS measurement (d)

particle size distribution (Fig. S3). Therefore, $\tau_{2}$ is likely due to the $\pi^{*}-\pi$ transition of the aromatic cores (i.e. $\mathrm{sp}^{2}$ skeleton) of GQDs and possess quantum confinement feature and therefore, have high correlation with particle size distribution, is responsible for the blue shift of the static PL as shown in Figure $1 \mathrm{a}$ and $\mathrm{b}$.

As for $\tau_{3}(10-14 \mathrm{~ns})$, in addition to its percentage rise after reduction, it presents a trend that its contribution increases from the left to the right of the PL spectra (Fig. 2 c). It suggests that this emissive component predominantly contributes to the red side of PL. From the XPS analysis (Fig. S7 and Table S1), during the removal of the oxygen contents, not only $\mathrm{C}=\mathrm{C}$ bonding but also the aliphatic carbon $\mathrm{C}-\mathrm{C}$ bonding increases, indicating the increase of disordered structures (i.e. defects and $\mathrm{sp}^{3}$ carbon). Figure $2 \mathrm{~d}$ also shows the change of atomic concentration of $\mathrm{C}-\mathrm{C}$ bonding prior and after the chemical reduction of the corresponding GQDs. The rise of $\mathrm{C}-\mathrm{C}$ bonding suggests the rise in composition of disordered structures, which coincides with the increase of $\tau_{3}$ of post chemical reduction. Therefore, we hypothesize that the disordered structures may be responsible for $\tau_{3}$; In particular, the disordered structures on GQDs may induce mid-gap energy states in between the $\pi^{*}-\pi$ gap allowing electrons to relax on these energy states (i.e. $\pi^{*}$ relaxation states $-\pi$ transitions) first before returning to ground state thereby causing bathochromic broadening of the PL spectrum. As $\tau_{3}$ is related to $\pi^{*}$-relaxation states- $\pi$ transitions, the associated PL components are also sensitive to the change of particle size, namely it is also responsible for the blue shift of the static PL as shown in Figure $1 \mathrm{a}$ and $\mathrm{b}$. Therefore, the PL components associated with both $\tau_{2}$ and $\tau_{3}$ are the source of blue shift as shown in Figure $1 \mathrm{a}$ and $\mathrm{b}$.

Hence, from above discussion, a multiple emissive mechanism should be responsible for the PL of the oxygenated GQDs. Such a PL mechanism directly relates to the different structures existing in the oxygenated GQDs including the 


\section{ARTICLE}
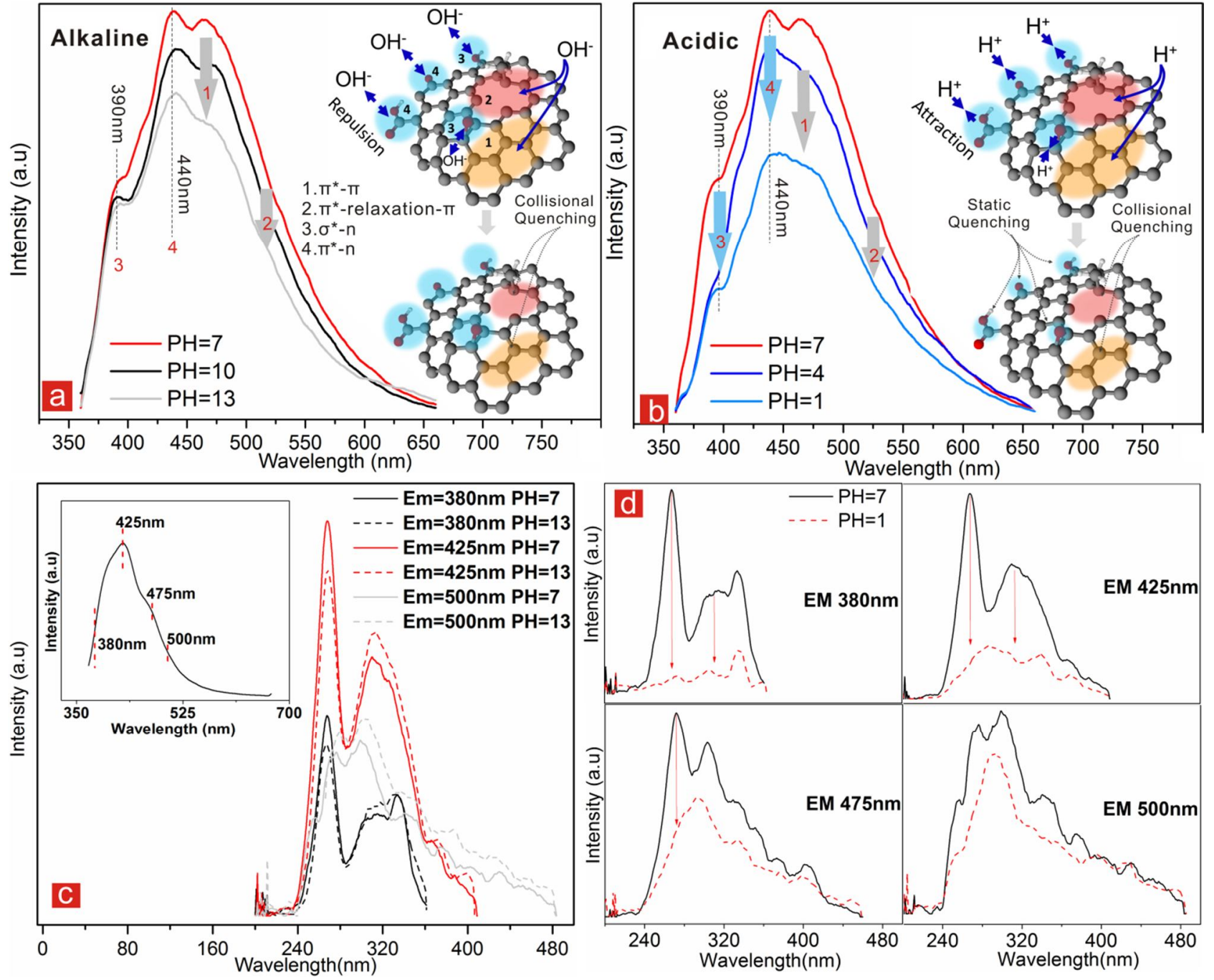

Figure 3. The response of G80 PL to PH under alkaline (a) and acidic (b) conditions respectively; Quenching tests of PLE of G210 under alkaline (c) and acidic (d) conditions. Insert in c is the static PL of G210 under 340nm excitation

aromatic core (i.e. $\mathrm{sp}^{2}$ skeleton), functional groups and disordered structures (i.e. defects and $\mathrm{sp}^{3}$ carbon).

\section{2 pH Influence on the PL of GQDs}

In order to further test the correlations between the PL components and the possible emissive sites of oxygenated GQDs, the $\mathrm{pH}$ influence on the PL of the as synthesized GQDs was also investigated. $\mathrm{pH}$ dependence is a widely reported phenomenon for oxygenated GQDs $9,10,25,26$. Figure S14 shows the PL spectra of G20, G80 and G210 under different $\mathrm{pH}$. Generally, the PL is quenched when the $\mathrm{pH}$ deviates from neutral for all of three GQDs, which is consistent with the existing literatures ${ }^{11,12,24,25}$. In this study, the $\mathrm{pH}$ response of
PL of GQDs was further evaluated in detail under acidic and alkaline conditions.

Figure $3 \mathrm{a}$ and $\mathrm{b}$ present the PL responses of G80 to alkaline and acidic conditions respectively. Under alkaline condition, the PL quenching (Fig. $3 \mathrm{a}$ ) is much less efficient on the left side than on the right side (i.e. red side). However, under acidic conditions, the PL appears to be quenched uniformly throughout the spectra. This suggests the quenching mechanisms by acidic conditions and alkaline conditions to the PL of GQDs are different. It is believed the PL quenching mechanism under alkaline condition is collisional quenching, in which the negatively charged functional groups effectively prevent the excessive $\mathrm{OH}^{-}$ions from accessing the sites where functional groups reside on the GQDs, therefore, they could 
only quench the $\pi-\pi^{*}$ transitions of the $\mathrm{sp}^{2}$ carbon area which they could access. As a result the blue side of the PL, particularly the spectrally invariable $390 \mathrm{~nm}$ (i.e. $n-\sigma^{*}$ emission) and $440 \mathrm{~nm}$ (i.e. $\mathrm{n}-\pi *$ emission) emissions as identified from last section, cannot be quenched as efficiently as the longerwavelength-side. However, the PL quenching mechanism under acidic conditions is most likely a mix of static and collisional quenching, in which due to the formation of nonradiative complexes between positive charged proton and negatively charged functional groups (e.g. $-\mathrm{COOH},-\mathrm{OH}$ ) both the $390 \mathrm{~nm}$ and $440 \mathrm{~nm}$ emission are statically quenched and, the $\pi-\pi^{*}$ transitions of the $\mathrm{sp}^{2}$ carbon area are collisionally quenched by the excessive protons in the environment ${ }^{27}$. In order to confirm the quenching mechanisms, the PLE spectra of G210 under strong acidic and alkaline conditions were evaluated as shown in Figure $3 \mathrm{c}$ and $\mathrm{d}$. Obviously, under alkaline condition $(\mathrm{pH}=13)$ only very limited variance from the neutral condition $(\mathrm{pH}=7)$ could be seen indicating the collisional feature of the corresponding quenching (Fig.3 c); however, under acidic condition ( $\mathrm{pH}=1$, Fig. $3 \mathrm{~d}$ ) the excitation peaks of the shortwavelength high energy emissions (i.e. emission $=380 \mathrm{~nm}$ or $425 \mathrm{~nm}$ ) were significantly reduced in contrast to the neutral condition, which is an indication of existence of nonradiative complexes ${ }^{27}$, and the long-wavelength low energy emissions (i.e. emission $=475 \mathrm{~nm}$ or $500 \mathrm{~nm}$ ) only present relative low degree of quench suggesting the collisional feature of the quenching.

Hence, combining the $\mathrm{pH}$-dependent $\mathrm{PL}$ and PLE, it is found that the two PL emissions at 390nm (i.e. $n-\sigma^{*}$ emission) and 440nm (i.e. $n-\pi *$ emission) of GQDs on the blue side of the spectra could be statically quenched by proton (i.e. $\mathrm{H}^{+}$) but barely sensitive to hydroxide ion (i.e. $\mathrm{OH}^{-}$); In contrast, the PL emissions (i.e. $\pi-\pi^{*}$ and $\pi^{*}$-relaxation states $-\pi$ transitions) on the red side of the spectra could be collisionally quenched by both proton (i.e. $\mathrm{H}^{+}$) and hydroxide ion (i.e. $\mathrm{OH}^{-}$). This is consistent with the PL mechanism as revealed by the static PL and TCSPC measurements in last section.

\subsection{Theoretical simulation}

In order to confirm the PL mechanisms from the experimental work, a density functional theory (DFT) simulation for systematic probing the effects of size of aromatic core, functional groups and disordered structures (i.e. defects and $\mathrm{sp}^{3}$ carbon) on the electronic structure of GQDs was performed (see supplementary information for the details of calculation). Briefly, we calculated the electronic structures of pristine GQDs (pGQDs, i.e. GQDs without any oxygen contents and disordered structures but only the hydrogen passivation on the edge), and oxidized GQDs (oGQDs, i.e. GQDs containing four types of oxygen functional groups (i.e. $\mathrm{OH}, \mathrm{C}-\mathrm{O}-\mathrm{C}, \mathrm{C}=\mathrm{O}$ and $\mathrm{COOH}$ ) of different sizes (i.e. 7 rings and 12 rings). In addition, a defective GQD (dGQD) was obtained by converting one carbon atom of the 7ring pGQD model into a methyl group creating both a defect and $\mathrm{sp}^{3}$ structure. The structures of the established models were based on the XPS characterization of as synthesized GQDs in this study. Specifically, the oxidized GQDs models possess $\mathrm{C} / \mathrm{O}$ ratios of $\sim 3$ similar to those of assynthesized GQDs in the experiments as revealed by the XPS data. Similar approach was also adopted for DFT simulation of GO for ensuring the representativeness of the constructed model to the experiment ${ }^{21}$. Moreover, since the main reactions take place at the edge of GQDs, we arrange most of the functional groups on the edge (i.e. hydroxyl, carbonyl and carboxyl), except for the epoxy group which preferably resides on the basal plane ${ }^{28}$. Since the simulations were conducted using a plane-wave basis set (or periodic boundary conditions), each model was isolated in an appropriately sized super cell (see supplementary information for details) which introduce sufficient vacuum along each of the axis, ensuring that the impact of the periodical images on the final calculation was minimized. Figure 4 presents the energy-level diagrams (ELDs) and corresponding density of states (DOS) from the DFT simulation along with the structural optimized 7 ring models which were applied for the calculations (see Figure S15 for the simulation results for 12 ring models).

As represented by the red lines in the ELDs in Figure 4, the dGQD and oGQDs possess energy levels almost identical to highest occupied molecular orbital (HOMO) and lowest unoccupied molecular orbital (LUMO) of pGQDs, suggesting that the oxygen-containing functional groups do not alter the confined bandgap of the aromatic core. The as calculated bandgaps (energy differences between HOMO and LUMO) for 7-ring and 12-ring models are 2.89ev (Fig.4a) and 1.61ev (Fig. $\mathrm{S} 14)$ respectively. The reduction of bandgap from 7-ring to 12 ring GQDs shows the size effect (i.e. quantum confinement). This is consistent with the blue shift of the static PL emission spectra with the decrease of dot size (i.e. decrease of the size of aromatic core) as presented in Fig.1. Another indication from the degradation of bandgap with increase of size is that the bandgap is quite sensitive to the change of size. From 7-ring (lateral size $\sim 0.7 \mathrm{~nm}$ ) to 12 -ring (lateral size $\sim 0.9 \mathrm{~nm}$ ), the bandgap decreases by $1.28 \mathrm{ev}$. Hence, there is no surprise that the PL blue shift in Fig. 1 from $500 \mathrm{~nm}(2.48 \mathrm{ev})$ to $420 \mathrm{~nm}$ $(2.95 \mathrm{ev})$, an approximate $0.5 \mathrm{ev}$ change in emission energy could result from the change of particle size by $1 \mathrm{~nm}$ (i.e. from $\sim 1.9 \mathrm{~nm}(\mathrm{G} 20)$ to $\sim 0.91 \mathrm{~nm}(\mathrm{G} 210)$.

Moreover, it is notable that in the ELDs of dGQD and oGQDs, additional energy levels appeared, as represented by the dark lines. For dGQD, the additional energy levels lie within the gap of LUMO and HOMO (Fig. 4 b). For oGQDs, these additional energy states scatter within the energy realm from the bottom area of LUMO to the top area of HOMO (Fig. $4 \mathrm{c}$ and Fig. S15 b). The DOS data also suggest the preservation of confinement and generation of additional energy states from pGQDs to dGQD and oGQDs, as the shape of DOS are similar for $\mathrm{pGQDs}$. dGQD and oGQDs except for the fact that the DOS is zero within the confined band gap for pGQDs and non-zero for dGQDs and oGQDs.

To further understand the nature of those additional energy states of pGQDs, dGQD and oGQDs, we calculated the MLWFs (a solid state equivalent of the molecular orbitals in chemistry) of several typical energy states (HOMO and these as indicated by the grey band E1, E2, E3, E4 in Fig.4) for the 7 ring $\mathrm{pGQD}$ model and its corresponding dGQD and oGQD models, and visualized the results in Figure 5 and S16. As shown in Figure S16, the HOMO of pGQD, dGQD and oGQD are all the naturally hybridized carbon-carbon $\pi$ orbitals (along the edge of the rings), and the emergence of disordered structures and functional groups do not change them in any significant ways. Figure 5a shows the visualized MLWF of the mid-gap energy state (i.e. as marked with E1 in Fig. 4, eigenvalue $=-1.85 \mathrm{ev}$ ) of dGQD. The MLWF of the selected mid-gap energy level of dGQD reside mainly on the disordered area of the dGQDs and the major composition is irregularly hybridized $\pi$ orbitals. It indicates that the emergence of disordered structure (i.e. defects and $\mathrm{sp}^{3}$ carbon) induced structural deformation to the GQDs. The structural deformation 


\section{ARTICLE}

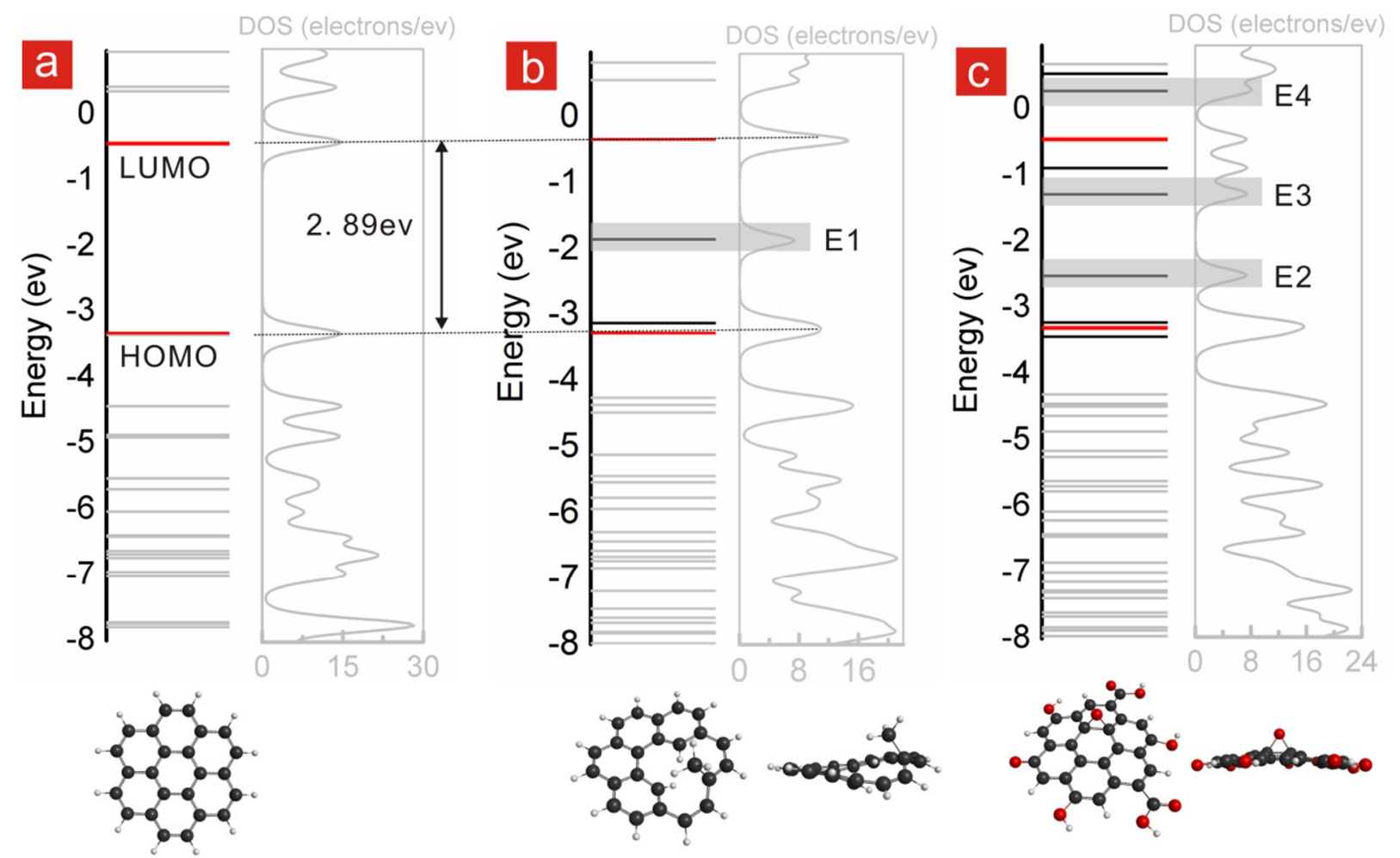

Figure 4. Energy level diagrams and DOS curves from the DFT calculation for 7 ring models: pGQDs (a), dGQD (b), and oGQDs (c); Cartoons under (a)-(c) represent the relaxed models used for each of the DFT calculation.

causes considerable distortion to the $\pi$ orbitals of the nearby carbon regions of the disordered structures. The consequence of such a distortion to the $\pi$ systems is the irregular hybridization of $\pi$ orbitals such as hybridizations among distorted $\mathrm{sp}^{2}$ bonds and long range hybridizations (i.e. the $\pi$ orbital hybridizations across the ring as shown in Fig. 5).

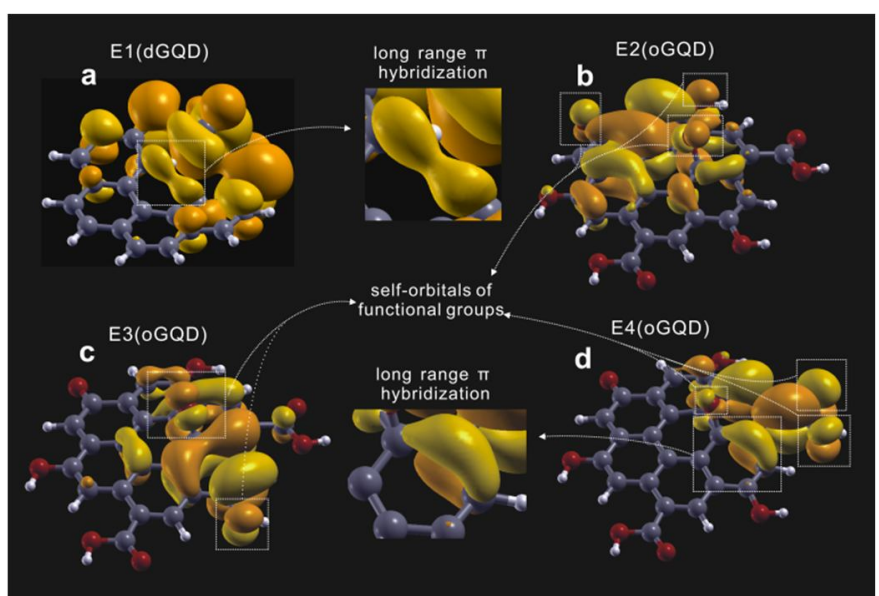

Figure 5. MLWFs of dGQD (a) and oGQD $(b \sim c)$ for energy states as indicated in Figure $4(\mathrm{E} 1 \sim \mathrm{E} 4)$
Figure $5 \mathrm{~b}$, c and d show three visualized MLWFs of the three typical (i.e. as marked with E2, E3, E4, in Fig. 4, eigenvalues are: $\mathrm{E} 2=-3.22 \mathrm{ev}, \mathrm{E} 3=-1.27 \mathrm{ev}, \mathrm{E} 4=-0.28 \mathrm{ev}$ ) of oGQDs. The MLWFs revealed that one important composition of those energy states are the self-orbitals of the functional groups (i.e. $\sigma, \pi$ and $\mathrm{n}$ orbitals of $\mathrm{OH}, \mathrm{C}-\mathrm{O}-\mathrm{C}, \mathrm{C}=\mathrm{O}$ and $\mathrm{COOH}$ ). We believe these self-orbitals of functional groups are the major source of functional group dominated emissions as revealed in sections 3.1 and 3.2 (i.e. $390 \mathrm{~nm}$ and $440 \mathrm{~nm}$ emissions in Fig.1 and Fig.3 ). The emission from self-orbitals of functional groups via transitions like $n-\sigma^{*}$ and $n-\pi^{*}$ has been reported for graphene oxide as well $1^{29}$. Apart from contributing self-orbitals, the attachment of functional groups also induces considerable deformation to the structure of GQDs, especially to the neighbouring areas where oxygen functional groups reside. Similar to the case of dGQD, the structural deformation induced by functional groups also causes the distortion of $\pi$ orbitals of the nearby carbon regions of the oxygen-containing functional groups, therefore, it also leads to irregular hybridizations of $\pi$ orbitals as shown in Figure 5. The energy states of such irregular hybridized $\pi$ orbitals caused either by disordered structures or functional groups are therefore named energy states induced by structural deformation (ESiD). These ESiD which possess energy levels in between the LUMO and HOMO of natural $\pi$ systems as indicated by the ELDs, could 
serve as the intermediate states between antibonding (i.e. $\pi^{*}$ ) and bonding states (i.e. $\pi$ ) of the natural $\pi$ systems thereby reducing the emission energy and prolonging the decay time. Judging from the structural optimized models (Fig. 6), the

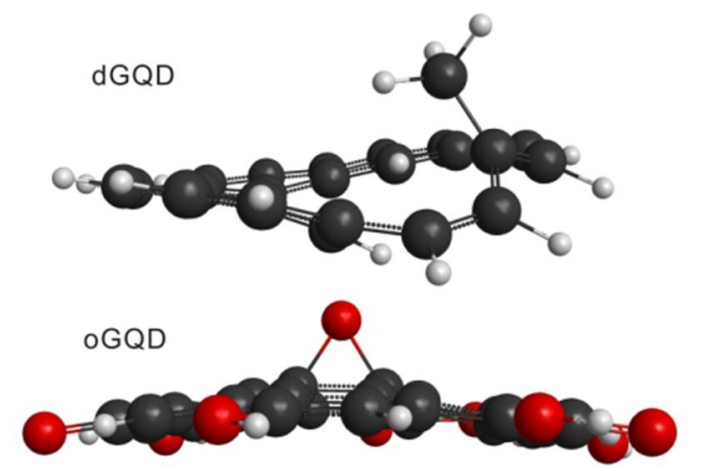

Figure 6. Structural optimized model for dGQD and oGQD

degree of structural deformation induced by disordered structures is in fact much more serious than the oxygen functional groups, which suggests that the disordered structures are more effective than functional groups in providing relaxation states. Therefore, from the MLWFs, the nature of the mid-gap energy states as estimated in section 3.1 is revealed. It is also learnt that these energy states could not just be caused by the disordered structures but also functional groups. Nevertheless the disordered structures are more effective in providing the mid-gap energy states than functional groups. This could explain why the rise of $\mathrm{C}-\mathrm{C}$ bonding after reduction could lead to the increase of $\tau 3$ as presented in Figure 2. We also proposed one of the possible electron transition path ways involving the ESiD in Figure S17.

Lastly, as mentioned before, the oGQD models of this study were established based on the XPS characterization of as synthesized GQDs in this study, in order to confirm the understanding about the roles of oxygenated functional groups could be generalized. We constructed an extra 7 ring model with a different distribution of functional groups (i.e. varied quantity of individual functional group and positions on the aromatic core of GQD) from the 7 ring model discussed above. The simulated results were presented in Figure S18. Despite the variation in distribution of functional groups, the simulated results are similar if not identical to the 7 ring oGQD model discussed above, which also suggest the dual roles of functional groups, namely contributing self-orbitals and inducing irregular hybridizations of $\pi$ orbitals of the aromatic core. Therefore, it suggests the dual roles of oxygen functional groups revealed in this research is applicable to GQDs with different distribution of oxygen-containing functional groups.

Hence, the theoretical simulation agrees well with the experimental results, revealing that the aromatic core, oxygenated functional groups and disordered structures (i.e. defects and $\mathrm{sp}^{3}$ carbon) all can be involved in the PL emission of oxygenated GQDs. Specifically, the oxygenated functional groups appear to possess two functions: one is to introduce the self-orbitals of functional groups $(n, \pi, \sigma)$ which are able to contribute emissive components with fixed positions on the PL spectrum through electronic transitions from anti-bonding states $\left(\pi^{*}, \sigma^{*}\right)$ to the non-bonding $(n)$ states; the other is to cause structural deformation to the GQDs. The disordered structures (i.e. defects and $\mathrm{sp}^{3}$ carbon) are also able to induce structural deformation to GQDs and they are more efficient to do so than the functional groups. The structural deformation caused by either functional groups or disordered structures leads to irregular hybridized $\pi$ orbitals with energy states (ESiDs) in between the confined $\pi-\pi^{*}$ gap of the aromatic core of dots. These orbitals could serve as the intermediate relaxation orbitals for the electrons from antibonding states $\left(\pi^{*}\right)$ to the bonding states $(\pi)$ of the aromatic core of the GQDs, contributing to the red side of the broadened emission that has prolonged PL lifetime.

\subsection{Proposed PL mechanism for oxygenated GODs}

Piecing together the discussions above, a possible emission mechanism for oxygenated GQDs was proposed as shown in Figure 7. The PL of oxygenated GQDs is an integration of the emissions of aromatic core and functional groups. Aromatic

core could contribute to the overall PL through $\pi^{*} \rightarrow \pi$ and $\pi^{*} \rightarrow \mathrm{ESiD} \rightarrow \pi$ transitions. The emissions of aromatic core possess quantum confinement feature (or 'size effect'), namely, the smaller the core size, the larger the bandgap and, the higher the emission energy is. Functional groups contribute to the overall PL through transitions such as $\pi^{*} \rightarrow \mathrm{n}, \sigma^{*} \rightarrow \mathrm{n}$. Different from the aromatic core emissions, the energy of emissions of functional groups are not influenced by the size of GQDs, therefore, the positions of PL components does not shift with the change of size. For the as-synthesized oxygenated GQDs in this research, the PL components by functional groups reside on the blue side of the core emissions. The ESiD are induced not only by disordered structures (i.e. defects and sp3 carbon) but also functional groups. Hence, the functional groups play duo roles in the PL of oxygenated GQDs.

\section{Conclusions}

Based on direct experimental evidences enabled by a sizetunable GQDs synthesis and supported by first-principle computation, we provided a detailed account on the contribution of aromatic cores, functional groups and disorder structures (i.e. defects and $\mathrm{sp}^{3}$ carbon) on the PL of GQDs. The key findings of this study can be summarised into: this study revealed that the functional groups play duo roles in the PL of GQDs, 1) they enable $\pi^{*} \rightarrow \mathrm{n}$ and $\sigma^{*} \rightarrow \mathrm{n}$ transitions, resulting in molecular type of PL components, spectrally invariable with the change of particle size or excitation energy; 2) similar to defects and $\mathrm{sp}^{3}$ carbon, they also induce structural deformation to the aromatic core, leading to irregular hybridization of the $\pi$ system providing mid-gap states (i.e. ESiD), allowing $\pi^{*} \rightarrow$ $\mathrm{ESiD} \rightarrow \pi$ transitions which cause bathochromic broadening of the integrated PL spectra. Therefore, we consider the observed PL spectra is an integration of emissions from aromatic core through both $\pi^{*} \rightarrow \pi$ and $\pi^{*} \rightarrow \mathrm{ESiD} \rightarrow \pi$ transitions which possess quantum confinement feature, and emissions from functional groups through $\pi^{*} \rightarrow \mathrm{n}$ and $\sigma^{*} \rightarrow \mathrm{n}$ transitions which spectrally invariable with the change of particle size or excitation energy. This mechanism, when generalised, will have significant value to the development of graphene-based optical materials for a broad range of applications ${ }^{20}$.

\section{Acknowledgements}

S.W. acknowledges the support of a Griffith International Postgraduate Scholarship (GIPS) and a CSIRO OCE top-up scholarship. Q.L. wishes to thank a Griffith University Research Infrastructure Grant (GURIP). The authors wish to 


\section{ARTICLE}
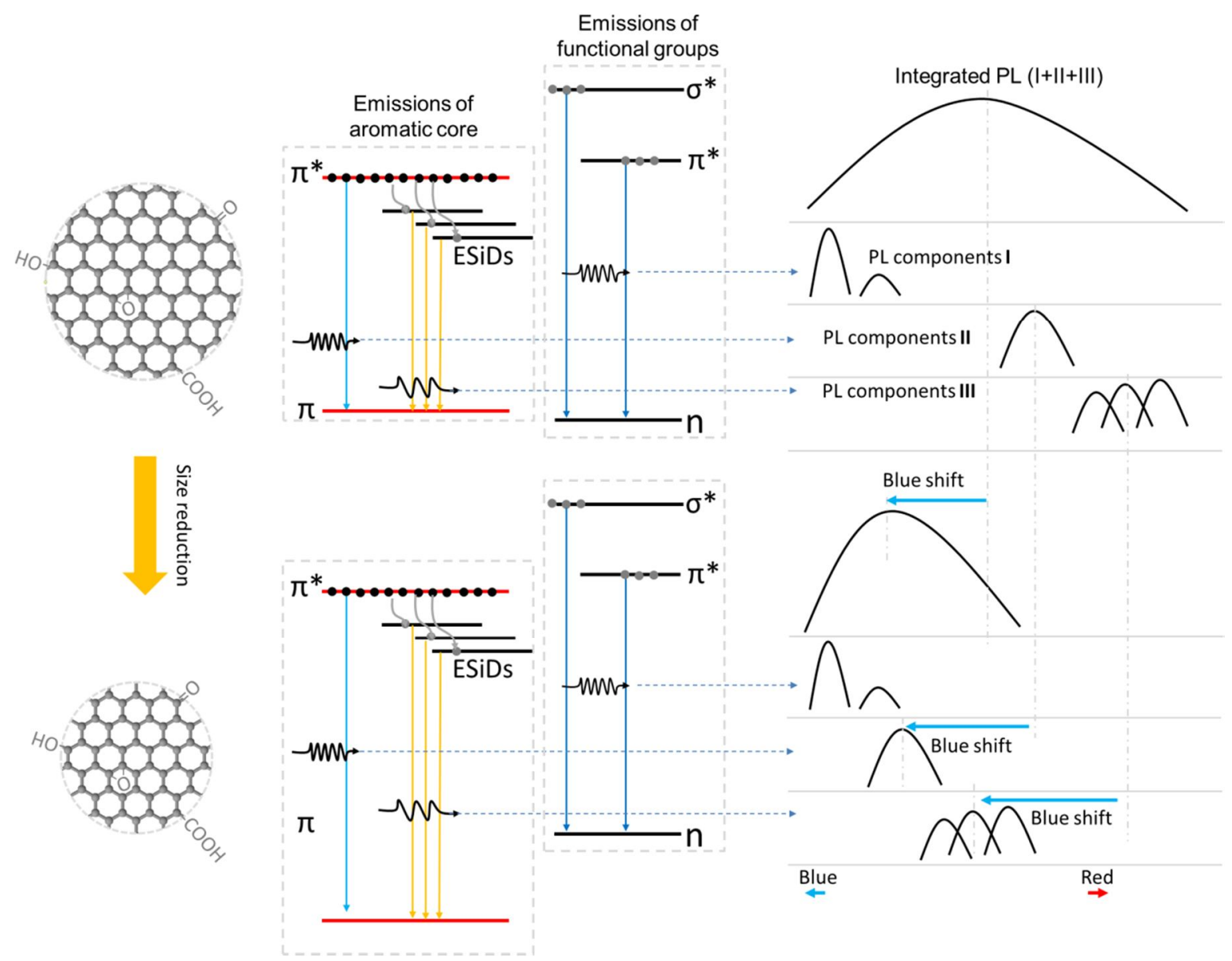

Figure 7. Schematic representation of the PL mechanism of oxygenated GQDs containing emissions of aromatic core and emissions of functional groups.

thank the Centre for Microscopy and Microanalysis at the University of Queensland for the characterization facilities andsupport, and Mr. Tak H. Kim for performing the time resolved photoluminescence spectroscopy measurement.

\section{Notes and references}

a Queensland Miro- and Nanotechnology Centre, Griffith University, Nathan Campus, Brisbane, QLD 4111, Australia.

${ }^{b}$ School of Engineering (Environmental), Griffith University, Nathan Campus, Brisbane, QLD 4111, Australia.

${ }^{c}$ CSIRO Materials Science and Engineering, -Gate 5, Normanby Road, Clayton, VIC 3168, Australia.

${ }^{d}$ Department of Chemistry \& Laboratory of Advanced Materials, Fudan University, Shanghai, 200433, P.R. China.

$\dagger$ Electronic Supplementary Information (ESI) available: [details of any supplementary information available should be included here]. See DOI: $10.1039 / \mathrm{b} 000000 \mathrm{x} /$
K. A. Ritter and J. W. Lyding, Nat Mater, 2009, 8, 235-242.

L. A. Ponomarenko, F. Schedin, M. I. Katsnelson, R. Yang, E. W. Hill, K. S. Novoselov and A. K. Geim, Science, 2008, 320, 356358.

W. L. Wang, S. Meng and E. Kaxiras, Nano Lett, 2008, 8, 241245.

Z. Z. Zhang and K. Chang, Phys Rev B, 2008, 77.

H. Q. Shi, A. S. Barnard and I. K. Snook, Journal of Materials Chemistry, 2012, 22, 18119-18123.

L. L. Li, G. H. Wu, G. H. Yang, J. Peng, J. W. Zhao and J. J. Zhu, Nanoscale, 2013, 5, 4015-4039.

Z. F. Wang, H. D. Zeng and L. Y. Sun, J Mater Chem C, 2015, 3, 1157-1165.

L.-L. Li, J. Ji, R. Fei, C.-Z. Wang, Q. Lu, J.-R. Zhang, L.-P. Jiang and J.-J. Zhu, Advanced Functional Materials, 2012, 22, 29712979.

D. Pan, J. Zhang, Z. Li and M. Wu, Advanced materials, 2010, 22 , 734-738.

J. Peng, W. Gao, B. K. Gupta, Z. Liu, R. Romero-Aburto, L. Ge, L. Song, L. B. Alemany, X. Zhan, G. Gao, S. A. Vithayathil, B. A. Kaipparettu, A. A. Marti, T. Hayashi, J. J. Zhu and P. M. Ajayan, Nano Lett, 2012, 12, 844-849. 
11. R. Q. Ye, C. S. Xiang, J. Lin, Z. W. Peng, K. W. Huang, Z. Yan, N. P. Cook, E. L. G. Samuel, C. C. Hwang, G. D. Ruan, G. Ceriotti, A. R. O. Raji, A. A. Marti and J. M. Tour, Nat Commun, 2013, 4.

12. S. J. Wang, Z. G. Chen, I. Cole and Q. Li, Carbon, 2015, 82, 304313.

13. Y. Q. Dong, H. C. Pang, S. Y. Ren, C. Q. Chen, Y. W. Chi and T. Yu, Carbon, 2013, 64, 245-251.

14. B. Mandal, S. Sarkar and P. Sarkar, Journal of Nanoparticle Research, 2012, 14.

15. M. Ezawa, Physica E, 2008, 40, 1421-1423.

16. G. Eda, Y. Y. Lin, C. Mattevi, H. Yamaguchi, H. A. Chen, I. S. Chen, C. W. Chen and M. Chhowalla, Advanced materials, 2010, 22, 505-509.

17. S. Zhu, J. Zhang, C. Qiao, S. Tang, Y. Li, W. Yuan, B. Li, L. Tian, F. Liu, R. Hu, H. Gao, H. Wei, H. Zhang, H. Sun and B. Yang, Chemical communications, 2011, 47, 6858.

18. F. Liu, M.-H. Jang, H. D. Ha, J.-H. Kim, Y.-H. Cho and T. S. Seo, Advanced materials, 2013, 25, 3657-3662.

19. S. Zhu, J. Zhang, S. Tang, C. Qiao, L. Wang, H. Wang, X. Liu, B. Li, Y. Li, W. Yu, X. Wang, H. Sun and B. Yang, Advanced Functional Materials, 2012, 22, 4732-4740.

20. K. P. Loh, Q. L. Bao, G. Eda and M. Chhowalla, Nat Chem, 2010, 2, 1015-1024.

21. J. Shang, L. Ma, J. Li, W. Ai, T. Yu and G. G. Gurzadyan, Scientific reports, 2012, 2, 792.

22. C. T. Chien, S. S. Li, W. J. Lai, Y. C. Yeh, H. A. Chen, I. S. Chen, L. C. Chen, K. H. Chen, T. Nemoto, S. Isoda, M. W. Chen, T. Fujita, G. Eda, H. Yamaguchi, M. Chhowalla and C. W. Chen, Angew Chem Int Edit, 2012, 51, 6662-6666.

23. S. J. Zhu, Y. B. Song, X. H. Zhao, J. R. Shao, J. H. Zhang and B. Yang, Nano research, 2015, 8, 355-381.

24 L. B. Tang, R. B. Ji, X. K. Cao, J. Y. Lin, H. X. Jiang, X. M. Li, K. S. Teng, C. M. Luk, S. J. Zeng, J. H. Hao and S. P. Lau, Acs Nano, 2012, 6, 5102-5110.

25. D. Pan, L. Guo, J. Zhang, C. Xi, Q. Xue, H. Huang, J. Li, Z. Zhang, W. Yu, Z. Chen, Z. Li and M. Wu, Journal of Materials Chemistry, 2012, 22, 3314.

26. J. Shen, Y. Zhu, X. Yang and C. Li, Chemical communications, 2012, 48, 3686-3699.

27. J. R. Lakowicz, Principles of fluorescence spectroscopy, Plenum Press, New York, 1983.

28. D. Chen, H. B. Feng and J. H. Li, Chem Rev, 2012, 112, 60276053.

29. M. Li, S. K. Cushing, X. Zhou, S. Guo and N. Wu, Journal of Materials Chemistry, 2012, 22, 23374. 